\title{
Editorials
}

\section{The utility of hemoglobin based oxygen carriers (HBOC) - can animal studies help?}

Richard I. Hall MD FRCPC FCCP

I $\mathrm{N}$ this issue of the Journal, Freitag et al. ${ }^{1}$ report their results of the effect of a hemoglobin based oxygen carrier (HBOC-201-Hemopure ${ }^{\mathrm{TM}}$ ) on augmentation of tissue oxygenation following isovolemic hemodilution. They have determined that, in their dog model, liver tissue oxygenation can be further improved by using HBOC-201 in a situation where physiological reserves are near their limits. What are the implications of their findings for the practicing anesthesiologist?

To put their findings into context, we must first review what the physiological consequences of isovolemic hemodilution (as used in their study) are as opposed to those which occur during unresuscitated hemorrhage. In the latter, as hemorrhage progresses, hemoglobin levels fall and to preserve homeostatis, cardiac output falls and systemic vascular resistance increases. Tissue perfusion is impaired leading to increased ischemia - particularly in the splanchnic bed. The clinical picture of cool extremities and reduced urine output will be familiar to any practitioner dealing with trauma patients.

In contrast, in the setting of isovolemic hemodilution (i.e., sufficient resuscitation with crystalloids or colloids so as to maintain intravascular volume albeit with a reduced hemoglobin level), the situation is somewhat different. As demonstrated by Freitag et al., ${ }^{1}$ systemic vascular resistance falls, cardiac output increases, and blood pressure may fall. In either case, oxygenation is preserved, at least initially, by increasing oxygen extraction as the hemoglobin concentration falls. However, even this safety mechanism will fail if the loss of hemoglobin continues. In short, if hemoglobin is not replaced in a timely fashion, organ dysfunction and death will ensue. In an attempt to extend the interval between impaired tissue oxygenation and death, hemoglobin based oxygen carriers (HBOC) have been developed.

Initial attempts at investigating these agents were frustrated by the development of complications. Cell free hemoglobin dissociates from the natural configuration (a tetramer) to dimers. These dimers are excreted by the kidney but are of sufficient size to cause tubular obstruction and eventual renal failure. In an attempt to avoid this complication, a variety of modifications were employed, but the agents which have been investigated recently involve cross-linking of the hemoglobin and polymerization of some sort. ${ }^{2}$

Hemoglobin based oxygen carriers are not designed to replace blood. Their purpose is to provide an oxygen carrying solution which can act as a bridge to transfusion in situations where blood may not be readily available e.g., battlefield injuries or trauma in rural settings $s^{3}$ or in situations where bleeding is anticipated and transfusion avoidance (including intraoperative normovolemic hemodilution and red blood cell harvesting for later transfusion) is desired. ${ }^{4,5}$ Various animal models have been designed to explore the use of HBOCs for these indications. These models allow one to determine not just that HBOCs can improve systemic oxygenation, but also how well tissue oxygenation is achieved under hypovolemic or normovolemic conditions.

In rats, Torres Filho et al. demonstrated in a normovolemic hemodilution model that an HBOC (hemoglobin raffimer) acts similarly to stored blood to maintain global oxygenation. ${ }^{6}$ Hare et al. demonstrated preserved brain oxygenation during normovolemic hemodilution in rats with hemoglobin raffimer. ${ }^{7}$ Renal perfusion and function were preserved in rats utiliz- 
ing hemoglobin raffimer in a normovolemic anemia model. ${ }^{8}$ Standl et al. showed preservation of skeletal muscle oxygenation in dogs. ${ }^{9}$ Thus the data from Freitag et al. ${ }^{1}$ are consistent with observations previously made in normovolemic anemia and extends our understanding by employing a larger animal (dog) and a different HBOC (Hemopure). A common observation in all of these studies is an increase in blood pressure and in systemic vascular resistance. This observation has been attributed to the ability of hemoglobin to scavenge nitric oxide, a potent endogenous vasodilator, although an interaction with endothelin and $\alpha$-adrenergic receptors may play a role as well. ${ }^{10}$

In contrast to the normovolemic hemodilution situation, the use of HBOCs for resuscitation from hypovolemia suggests there may be differences between the normovolemic and hypovolemic state in the ability of these compounds to maintain tissue perfusion perhaps due to nitric oxide induced vasoconstriction. In hypovolemic swine utilizing HBOC-201 (Hemopure) for resuscitation, liver $\mathrm{PO}_{2}$ was lower than in contols ${ }^{11}$ and York et al. observed hepatocellular injury with a similar model. ${ }^{12}$ Using diaspirin cross-linked hemoglobin in a hypovolemic swine model, van Iterson et al. observed preservation of gut oxygenation but an inability to preserve heart oxygenation ${ }^{13}$ whereas Pape et al. observed that regional perfusion to the pancreas and kidneys was impaired. ${ }^{14}$ Using HBOC-201, Lee et al. demonstrated preservation of brain oxygenation in dogs. ${ }^{15}$

The above studies serve to alert us about potential differences in the performance of the different HBOCs under normovolemic or hypovolemic situations. Unfortunately, none of these agents are available for clinical use in North America. Despite promising early results, the development of diaspirin cross-linked hemoglobin ${ }^{16,17}$ and hemoglobin-raffimer (Hemolink) ${ }^{18-20}$ have been terminated because of an adverse risk-benefit profile. A hemoglobin based oxygen carrier (Hemopure) is available in South Africa and an application is before the Food and Drug Administration for approval in the USA. Promising results have been published. .1,22 $^{2}$ Polymerized pyridoxilated hemoglobin (Polyheme) is still being investigated in trauma patients. A recombinant hemoglobin is in early investigational studies. ${ }^{23}$ Whether any of these will be available for clinical use is unknown. However, studies such as that of Freitag et al. ${ }^{1}$ provide information which may alert us as to what potential complications we should look for, and the conditions under which they might occur when (or if) a HBOC does become available.

\section{L'utilité des trans-} porteurs d'oxygène à base d'hémoglobine (HBOC) - les études animales peuvent-elles aider?

Dans le présent numéro du Journal, Freitag et coll. ${ }^{1}$ présentent l'effet d'un transporteur d'oxygène à base d'hémoglobine (HBOC-201-Hemopure ${ }^{\mathrm{TM}}$ ) sur l'augmentation de l'oxygénation tissulaire à la suite d'hémodilution isovolémique. Ils ont trouvé que, chez leur modèle canin, l'oxygénation tissulaire du foie peut être encore supérieure en utilisant HBOC201 quand les réserves physiologiques sont presque à la limite. Quelles sont les implications de leurs résultats sur la pratique de l'anesthésie ?

Revoyons d'abord les conséquences physiologiques de l'hémodilution isovolémique, en fonction de son utilisation dans l'étude, par opposition à celles qui surviennentpendant une hémorragie non traitée. Dansce dernier cas, à mesure que l'hémorragie progresse, les niveaux d'hémoglobine chutent et, pour préserver l'homéostase, le débit cardiaque diminue et la résistance vasculaire générale augmente. La perfusion tissulaire est atteinte, ce qui augmente l'ischémie - dans la circulation splanchnique en particulier. Des extrémités froides et une miction réduite constituent un portrait clinique familier aux praticiens traitant des polytraumatisés.

A l'opposé, la situation est un peu différente en cas d'hémodilution isovolémique, c'est-à-dire d'une réanimation suffisante avec des cristalloïdes ou des colloïdes pour maintenir le volume intravasculaire, bien qu'avec un niveau d'hémoglobine réduit. Freitag et coll. ${ }^{1}$ ont montré que la résistance vasculaire générale tombe, le débit cardiaque augmente et la tension artérielle peut baisser. Dans les deux cas, l'oxygénation est préservée, du moins au début, par une plus importante extraction d'oxygène au moment de la baisse de concentration d'hémoglobine. Cependant, ce mécanisme de sécurité va aussi flancher si la perte d'hémoglobine se poursuit. Bref, si l'hémoglobine n'est pas remplacée à temps, une dysfonction organique et la mort vont survenir. C'est pour prolonger le temps d'intervention après la baisse de l'oxygénation tissulaire que les trans- 
porteurs d'oxygène à base d'hémoglobine (HBOC) ont été développés.

Les premières explorations de ces agents ont été frustrantes à cause des complications. Le tétramère, configuration naturelle de l'hémoglobine acellulaire, se dissocie en dimères. Ces dimères sont éliminés par le rein mais sont de taille suffisante pour causer une obstruction tubulaire et une défaillance rénale par la suite. Pour éviter cette complication, diverses modifications ont été employées, mais l'élaboration des agents testés récemment implique la réticulation de l'hémoglobine et la polymérisation d'une façon quelconque. ${ }^{2}$

Les transporteurs d'oxygène à base d'hémoglobine ne peuvent remplacer le sang. Ils fournissent une solution qui transporte de l'oxygène pouvant agir comme une transition vers la transfusion dans des situations où le sang n'est pas immédiatement accessible, comme en médecine de guerre ou dans les cas de traumatisme en milieu rural ${ }^{3}$ ou dans des situations où une hémorragie est prévisible mais la transfusion n'est pas souhaitée, ce qui comprend l'hémodilution normovolémique peropératoire et le prélèvement de culots globulaires pour une transfusion ultérieure., ${ }^{4,5}$ Divers modèles animaux ont été développés pour explorer l'usage des HBOC dans ces situations. Ces modèles permettent de déterminer non seulement que les HBOC peuvent améliorer l'oxygénation générale, mais aussi quelle qualité d'oxygénation tissulaire est fournie dans des conditions hypovolémiques ou normovolémiques.

Torres Filho et coll. ont montré, dans un modèle d'hémodilution normovolémique chez les rats, qu'une HBOC (raffimère d'hémoglobine) agit de façon similaire au sang d'un culot globulaire pour maintenir l'oxygénation générale. ${ }^{6}$ Hare et coll. ont montré que l'oxygénation cérébrale est préservée avec un raffimère d'hémoglobine pendant l'hémodilution normovolémique chez des rats. ${ }^{7}$ La perfusion et la fonction rénales ont été préservées chez des rats avec un raffimère d'hémoglobine chez un modèle d'anémie normovolémique. ${ }^{8}$ Standl et coll. ont montré la préservation de l'oxygénation des muscles squelettiques chez des chiens. ${ }^{9}$ Les données de Freitag et coll. ${ }^{1}$ correspondent donc aux observations faites précédemment pendant l'anémie normovolémique et elles ajoutent à nos connaissances en employant un animal plus gros, le chien, et une HBOC différente, l'Hemopure. Toutes ces études partagent une augmentation de la tension artérielle et de la résistance vasculaire générale. Ce résultat est attribué à la capacité de l'hémoglobine de fixer l'oxyde nitrique, un vasodilatateur endogène puissant, malgré qu'une interaction avec l'endothéline et les récepteurs $\alpha$-adrénergiques puissent aussi jouer un rôle. ${ }^{10}$
À la différence de ce qui se passe lors d'hémodilution normovolémique, l'HBOC utilisée après hypovolémie laisse croire que les différents composés ne maintiennent pas la perfusion tissulaire de la même manière. Avec l'usage de HBOC-20l (Hemopure) pour la réanimation chez le porc hypovolémique, la $\mathrm{PO}_{2}$ hépatique a été plus faible que chez les témoins ${ }^{12}$ et York et coll. ont observé une lésion hépatocellulaire chez un modèle similaire. ${ }^{12}$ Avec la diaspirine en liaison avec l'hémoglobine chez un modèle porcin d'hypovolémie, van Iterson et coll. ont noté la préservation de l'oxygénation intestinale, mais non de l'oxygénation cardiaque ${ }^{13}$ alors que Pape et coll. ont observé une atteinte de la perfusion régionale vers le pancréas et les reins. ${ }^{14}$ Lee et coll. ont trouvé que l'oxygénation cérébrale est préservée chez des chiens ${ }^{15}$ avec HBOC-201.

Les études citées nous apprennent que des différences de performance sont possibles selon l'HBOC utilisée dans des conditions normovolémiques ou hypovolémiques. Malheureusement, aucune n'est disponible pour usage clinique en Amérique du Nord. Malgré des premiers résultats prometteurs, le développement de diaspirine liée à l'hémoglobine ${ }^{16,17}$ et d'un raffimère d'hémoglobine, Hemolink, ${ }^{18-20}$ a été arrêté faute d'un rapport risque/bénéfice favorable. Le transporteur d'oxygène à base d'hémoglobine (Hemopure) est disponible en Afrique du Sud et il y a une demande pendante devant la Food and Drug Administration pour approbation aux É-U. Des résultats encourageants ont été publiés. ${ }^{21,22}$ L'hémoglobine pyridoxilée polymérisée (Polyheme) est toujours à l'étude chez des polytraumatisés. Les premières études avec une hémoglobine recombinante sont en cours. ${ }^{23}$ On ne sait encore si l'un de ces composés sera accessible en clinique. Toutefois, des études comme celle de Freitag et coll. fournissent des informations qui peuvent nous préparer à rechercher certaines complications possibles et les conditions dans lesquelles elles peuvent survenir quand, ou si, une HBOC devient disponible.

\section{References}

1 Freitag M, Standl TG, Gottschalk A, et al. Enhanced central organ oxygenation after application of bovine cell-free hemoglobin HBOC-201. Can J Anesth 2005; 52: 904-14.

2 Carmichael FJ. Recent developments in hemoglobinbased oxygen carriers--an update on clinical trials. Transfus Apheresis Sci 2001; 24: 17-21.

3 Arnoldo BD, Minei JP. Potential of hemoglobin-based oxygen carriers in trauma patients. Curr Opin Crit Care 2001; 7: 431-6. 
4 Greenburg $A G$, Kim $H W$. Hemoglobin-based oxygen carriers. Crit Care 2004; 8(Suppl 2): S61-4.

$5 \mathrm{Wahr} J A$. Clinical potential of blood substitutes or oxygen therapeutics during cardiac surgery. Anesthesiol Clin North America 2003; 21: 553-68.

6 Torres Filho IP, Spiess BD, Barbee RW, Ward KR, Oldenhof J, Pittman RN. Systemic responses to hemodilution after transfusion with stored blood and with a hemoglobin-based oxygen carrier. Anesth Analg 2005; 100: 912-20.

7 Hare GM, Hum KM, Kim SY, Barr A, Baker AJ, Mazer $C D$. Increased cerebral tissue oxygen tension after extensive hemodilution with a hemoglobin-based oxygen carrier. Anesth Analg 2004; 99: 528-35.

8 Lieberthal W, Fubro R, Freedman JE, Toolan G, Loscalzo $J$, Valeri $C R$. O-raffinose cross-linking markedly reduces systemic and renal vasoconstrictor effects of unmodified human hemoglobin. J Pharmacol Exp Ther 1999; 288: 1278-87.

9 Standl T, Freitag M, Burmeister MA, Horn EP, Wilhelm $S, A m$ Esch JS. Hemoglobin-based oxygen carrier HBOC-201 provides higher and faster increase in oxygen tension in skeletal muscle of anemic dogs than do stored red blood cells. J Vasc Surg 2003; 37: 859-65.

10 Fitzpatrick CM, Savage SA, Kerby JD, Clouse WD, Kashyap VS. Resuscitation with a blood substitute causes vasoconstriction without nitric oxide scavenging in a model of arterial hemorrhage. J Am Coll Surg 2004; 199: 693-701.

11 Knudson MM, Lee S, Erickson V, Morabito D, Derugin $N$, Manley GT. Tissue oxygen monitoring during hemorrhagic shock and resuscitation: a comparison of lactated Ringer's solution, hypertonic saline dextran, and HBOC-201. J Trauma 2003; 54: 242-52.

12 York GB, Eggers JS, Smith DL, et al. Low-volume resuscitation with a polymerized bovine hemoglobinbased oxygen-carrying solution (HBOC-201) provides adequate tissue oxygenation for survival in a porcine model of controlled hemorrhage. J Trauma 2003; 55: 873-85.

13 van Iterson $M$, Siegemund $M$, Burbop $K$, Ince $C$. Hemoglobin-based oxygen carrier provides heterogeneous microvascular oxygenation in heart and gut after hemorrhage in pigs. J Trauma 2003; 55: 1111-24.

14 Pape A, Kleen M, Kemming G, Meisner F, Meier J, Habler $O$. Fluid resuscitation from severe hemorrhagic shock using diaspirin cross-linked hemoglobin fails to improve pancreatic and renal perfusion. Acta Anaesthesiol Scand 2004; 48: 1328-37.

15 Lee SK, Morabito D, Hemphill JC, et al. Small-volume resuscitation with HBOC-201: effects on cardiovascular parameters and brain tissue oxygen tension in an out-of-hospital model of hemorrhage in swine. Acad
Emerg Med 2002; 9: 969-76.

16 Schubert A, Przybelski RJ, Eidt JF, et al. Diaspirincrosslinked hemoglobin reduces blood transfusion in noncardiac surgery: a multicenter, randomized, controlled, double-blinded trial. Anesth Analg 2003; 97: 323-32.

17 Lamy ML, Daily EK, Brichant JF, et al. Randomized trial of diaspirin cross-linked hemoglobin solution as an alternative to blood transfusion after cardiac surgery. The DCLHb Cardiac Surgery Trial Collaborative Group. Anesthesiology 2000; 92: 646-56.

18 Hill SE, Gottschalk LI, Grichnik K. Safety and preliminary efficacy of hemoglobin raffimer for patients undergoing coronary artery bypass surgery. J Cardiothorac Vasc Anesth 2002; 16: 695-702.

19 Cheng DC, Mazer CD, Martineau R, et al. A phase II dose-response study of hemoglobin raffimer (Hemolink) in elective coronary artery bypass surgery. J Thorac Cardiovasc Surg 2004; 127: 79-86.

20 Greenburg AG, Kim HW; Hemolink Study Group. Use of an oxygen therapeutic as an adjunct to intraoperative autologous donation to reduce transfusion requirements in patients undergoing coronary artery bypass graft surgery. J Am Coll Surg 2004; 198: 373-83.

21 Sprung J, Kindscher JD, Wahr JA, et al. The use of bovine hemoglobin glutamer-250 (Hemopure) in surgical patients: results of a multicenter, randomized, single-blinded trial. Anesth Analg 2002; 94: 799-808.

22 Levy JH, Goodnough LT, Greilich PE, et al. Polymerized bovine hemoglobin solution as a replacement for allogeneic red blood cell transfusion after cardiac surgery: results of a randomized, double-blind trial. J Thorac Cardiovasc Surg 2002; 124: 35-42.

23 Raat NJ, Liu JF, Doyle MP, Burhop KE, Klein J, Ince C. Effects of recombinant-hemoglobin solutions rHb2.0 and rHbl.1 on blood pressure, intestinal blood flow, and gut oxygenation in a rat model of hemorrhagic shock. J Lab Clin Med 2005; 145: 21-32. 